\title{
Fructose dehydration to hydroxyl-methylfurfural in an immobilized catalytic microreactor
}

\author{
Aura Visan $^{1} \cdot$ Rob G. H. Lammertink ${ }^{1}$ (i) \\ Received: 29 December 2019 / Accepted: 17 February 2020 / Published online: 18 March 2020 \\ (C) The Author(s) 2020
}

\begin{abstract}
In this paper we report a microfluidic platform that allows for high temperature, high pressure conversion with inline spectroscopic measurement for a fast and accurate determination of both reaction rate constant and activation energy. The dehydration of fructose to hydroxyl-methylfurfural has been performed in this immobilized microreactor with both dense zirconia and porous titania layers, as a starting point to probe the potential of abundant metal oxide catalysts.
\end{abstract}

Keywords Microreactor · Catalysis · Fructose to HMF

\section{Introduction}

Catalysis is ever-present in industry with up to $90 \%$ of the reaction processes using catalysts. Most of these processes comprise fossil based feedstock which entails selective functionalization of apolar, unfunctionalized hydrocarbons. For well-known reasons, there is a global interest for more sustainable resources. The catalytic conversion of biomass waste is a promising alternative for chemicals, materials, and fuel production [1-3]. Especially for chemical synthesis, this route is challenging due to the richness and complexity of the chemical composition of biomass waste. While oil consists of hydrocarbons, biomass is high in oxygen content and consequently hydrophilic. Catalysts will have to fulfil completely different requirements in the case of biomass conversion, as the chemical conversion will entail selective defunctionalisation of polar, highly functionalized oxygenates.

Electronic supplementary material The online version of this article (https://doi.org/10.1007/s41981-020-00087-5) contains supplementary material, which is available to authorized users.

Rob G. H. Lammertink

r.g.h.lammertink@utwente.nl

Aura Visan

a.visan@utwente.nl

1 Soft Matter, Fluidics and Interfaces, University of Twente, Enschede, The Netherlands
Future bio-refineries need to produce high value bio based chemicals to be economically competitive. There are two possible strategies for approaching the market. The first is to aim for novel products with new and improved properties such is the case of 2,5-furan dicarboxylic acid which has the prospect of replacing terephthalic acid in the fabrication of PET [4]. In this context, markets need to develop. The second scenario is to aim for existing products that utilize existing infrastructure and for which there is already a mature market [5]. Both processing routes converge towards a few important platform molecules from which a myriad of end products can diverge. Dusselier identified that carbohydrates give access to a plethora of chemicals, including 5-hydroxymethylfurfural (HMF), levulinic acid (LA) and $\gamma$-valerolactone (GVL) [6-8].

HMF is a versatile and promising compound derived from carbohydrates. It can be used as a renewable intermediate for the production of polymers, fuels or solvents in the petrochemical industry. The last step in the synthesis of HMF is the dehydration of fructose (Fig. 1). Researchers have developed biocatalysts to produce fructose from cellulose [9], which is one of the major component of most plants and agriculture wastes [10]. An environmental-friendly process with a high fructose conversion rate is desirable for the overall utilization of biomass.

The challenge for the effective conversion of biomass is to develop catalysts for efficient conversion at low cost [11-14]. Group VIII metals, notably ruthenium, have shown to facilitate the hydrogenation step in liquefaction, but the cost of these precious metals is extremely high. Inexpensive 
Fig. 1 Reaction scheme for the dehydration of fructose to hydroxymethylfurfural

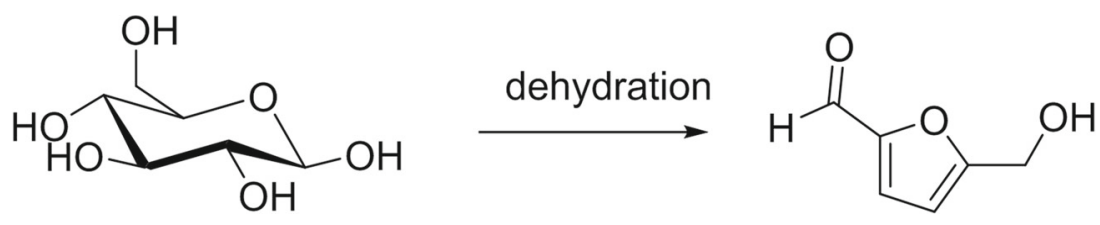

heterogeneous catalysts, such as zirconium dioxide and titanium dioxide are attracting increasingly more attention as alternatives [15]. Moreover, due to its high content in oxygen, biomass is typically processed in aqueous solutions or other polar solvents such as alcohols. These polar solvents at high temperatures and pressures and often at extreme $\mathrm{pH}$ challenge the stability of most catalysts. Among the few that can withstand these severe conditions are metals oxides.

Solid acids such as, phosphates [18] and chlorides [19, $20]$, but also plain metal oxides $[16,21]$ have been studied as catalysts in the dehydration of fructose. It has been proven that metal oxides increase their acid site density upon treatment with phosphoric acid which improves the dehydration from fructose to $\operatorname{HMF}[17,21]$. The treatment with phosphoric acid, esterifies - $\mathrm{OH}$ groups on the surface of $\mathrm{TiO}_{2}$ into $-\mathrm{O}-\mathrm{PO}\left(\mathrm{OH}_{2}\right)$ which increases the HMF selectivity $[11,17]$. What is more, $\mathrm{TiO}_{2}$ surfaces exhibit super - hydrophilicity during and after UV light exposure due to the formation of excess surface - $\mathrm{OH}$ groups. The combination of UV light and phosphoric acid treatment would increase the density of surface phosphate species, thereby enhancing its catalytic performance [11].

Microreactors form an attractive platform for kinetic investigations for heterogeneous catalysis. Their small dimensions provide a laminar flow profile and, consequently, a well-defined mass transport. They also allow for fast inline measurement without the need to quench the reaction for sample collection and analysis. The current project investigates one of the key reactions in the conversion of biomass waste, namely fructose to hydroxymethylfurfural (Fig. 1), in a microreactor device at elevated pressures and temperatures.

\section{Experimental}

\section{Chip fabrication}

The fabrication of microchannels and inlet/outlet holes in silicon was achieved using photolithography and deep reactive ion etching. Anodic bonding of the glass cover to silicon gives a very strong bond without the need of excessive heating which could affect the morphology of the immobilized catalyst. Having the channels in silicon is beneficial due to its high thermal conductivity and glass is the obvious choice to close the microreactor because it gives the possibility of UV irradiation necessary for the surface functionalization of the catalyst and the use of microscopic techniques for in-situ observations. The meandering channel (Fig. 2) is $500 \mu \mathrm{m}$ wide and $50 \mu \mathrm{m}$ deep and $18 \mathrm{~cm}$ long.

We have used two types of catalysts. A dense zirconia layer which was sputtered during the cleanroom fabrication and a porous titania which was wash coated after closing the reactor. The cleanroom fabrication steps include photolithography, opening the $\mathrm{SiO}_{2}$ mask, deep reactive ion etching of the microchannels, lift-off, second photolithography on the back, deep reactive ion etching the inlet and outlet holes, as well as the gap separating the heated area from the fluidic connections, again lift off, wet etching of the $\mathrm{SiO}_{2}$ mask, photolithography for the third time by spray coating the photoresist to homogeneously cover the 3D structures, sputtering the catalyst (see below), lift off in acetone bath, anodic bonding and dicing (Figure S1). The success of this patterning is due to the thick resist that is obtained by spray coating. The $5 \mu \mathrm{m}$ layer ensures that no edge connection takes place upon sputtering. The second type of microreactor does not require a third
Fig. 2 a Microreactor CAD design. b The actual microreactor with the sputtered $\mathrm{ZrO}_{2}$ layer (visible as the purple colour) a

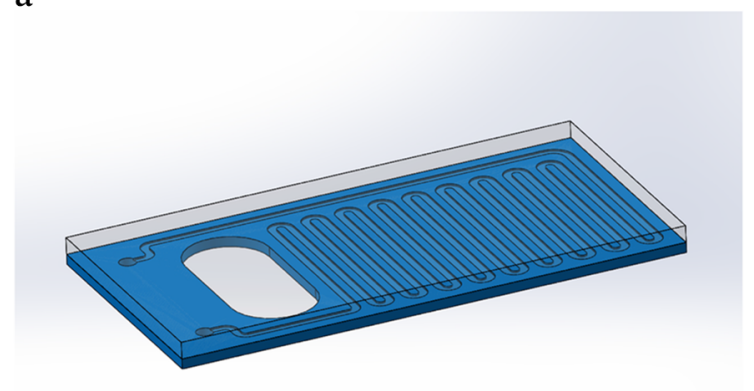

b

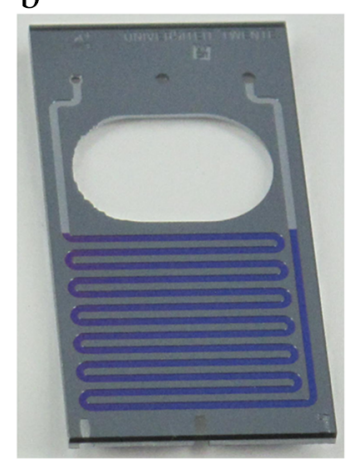


photolithography step and it was closed after deep reactive ion etching the back.

\section{Catalyst deposition and characterization}

Zirconium dioxide was deposited by reactive magnetron sputtering with a zirconium target using a dc power source. The film was sputtered for $40 \mathrm{~min}$ at $200 \mathrm{~W}$ using a reactor gas mixture of $92.5 \mathrm{vol} \% \mathrm{Ar}$ and $7.5 \mathrm{vol} \% \mathrm{O}_{2}$ at a process pressure of $5 \times 10^{-3}$ mbar. The layer was annealed for 4 $\mathrm{h}$ at $500^{\circ} \mathrm{C}$ in air. The heating and cooling rates were kept at $2{ }^{\circ} \mathrm{C} / \mathrm{min}$. High resolution scanning electron microscopy revealed a nonporous film with significant roughness. The elemental stoichiometry of the metal oxide was investigated using Energy Dispersive X-ray spectroscopy. The chemical composition was also confirmed by X-Ray Photoelectron Spectroscopy. The thickness and roughness were determined by Spectroscopic Ellipsometry using the method described by Visan et al. [22]. X-ray Diffraction was used to investigate the degree of crystallinity and detect the crystalline phases. The orientation of the crystallites was visualized by TEM.

The second microreactor was wash coated with a porous titania layer using a monodisperse commercial suspension (VP Disp. W 2730 X, Evonik). The suspension was used without dilution at the initial $30 \%$ (wt.) solid content. The aqueous dispersion is pumped through the microchannel and flushed afterwards at a constant displacement velocity to ensure a constant thickness along the channel. The resulting layer was sintered for $2 \mathrm{~h}$ at $500{ }^{\circ} \mathrm{C}$ in air. The heating and cooling rates were $2{ }^{\circ} \mathrm{C} / \mathrm{min}$. The quality of the suspension gave a homogeneous layer according to high resolution scanning electron microscopy. The narrow size distribution and absence of aggregates of the starting suspension was confirmed by light scattering measurements using a Zetasizer. The roughness and porosity was determined by spectroscopic ellipsometry [22]. The crystalline composition is provided by the manufacturer.

After sintering the wash coated layer, $\mathrm{TiO}_{2}$ was treated with phosphoric acid under UV. The microchannels were flushed with $1 \mathrm{M} \mathrm{H}_{3} \mathrm{PO}_{4}$ solution for $15 \mathrm{~min}$ at $50 \mu \mathrm{l} / \mathrm{min}$. While filled with $\mathrm{H}_{3} \mathrm{PO}_{4}$ solution, the microreactors were fixed under UV light (Dr. Gröbel UV light source HP$120,180 \mathrm{~mW} / \mathrm{cm}^{2}$ ) for 4 hours. After treatment, the microchannels were rinsed thoroughly with distilled water.

\section{Modular packaging}

The chipholder design is illustrated in Fig. 3. We placed the heating element inside the chipholder to keep a compact design. To limit the heating at the connection side, a part of the silicon between the connection zone and heated zone is

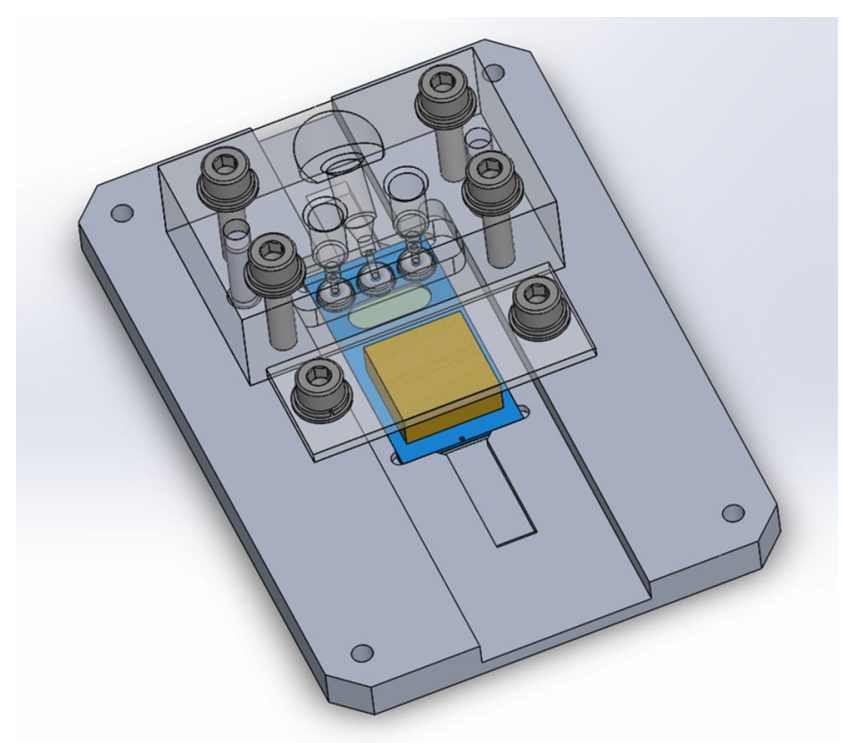

Fig. 3 Chipholder CAD design displaying the configuration of the TEC element

removed according to the design of Samuel Marre [23]. The temperature difference between the reaction zone and the port side has been assessed by using a second temperature sensor in order to check if active cooling is required on the compression side. The system proved to be leakage free until 50 bar. We opted for silicone o-rings due to their higher flexibility compared to Karlez. Karlez and Valco gave good sealing when newly installed.

The heating was performed locally on the reaction side using a Peltier element which allowed for accurate temperature control up to $200^{\circ} \mathrm{C}$. The chipholder has two separate top parts, the fluidic connection and the aluminum plate that pushes down the Peltier element which also works as a heat sink to ensure the heat flux through the element. This separate top prevents heat dissipation to the rest of the chipholder. The commercial temperature control system does not go higher than $120-150^{\circ} \mathrm{C}$ for thermoelectric (TEC) cooling elements, so individual components were put together in house. Two separate temperature measurements are possible. On the reaction side this is done with a Pt100 sensor that provides a very high accuracy in the order of $10^{-3}{ }^{\circ} \mathrm{C}$, while a NPT sensor monitors the compression side. The thermocouple tip is positioned very close to the microchannels inside a separate pocket. A 24 watt resistive heater can replace the Peltier within the same control unit to extend the temperature range up to $250^{\circ} \mathrm{C}$. The chipholder was fabricated entirely from PEEK in order to minimize the heat loss from the Peltier element. It also has a window on the glass side of the chip which allows microscopic observation. 
Fig. 4 Schematic illustration of the used setup, combining temperature and flow control with inline UV-Vis analysis

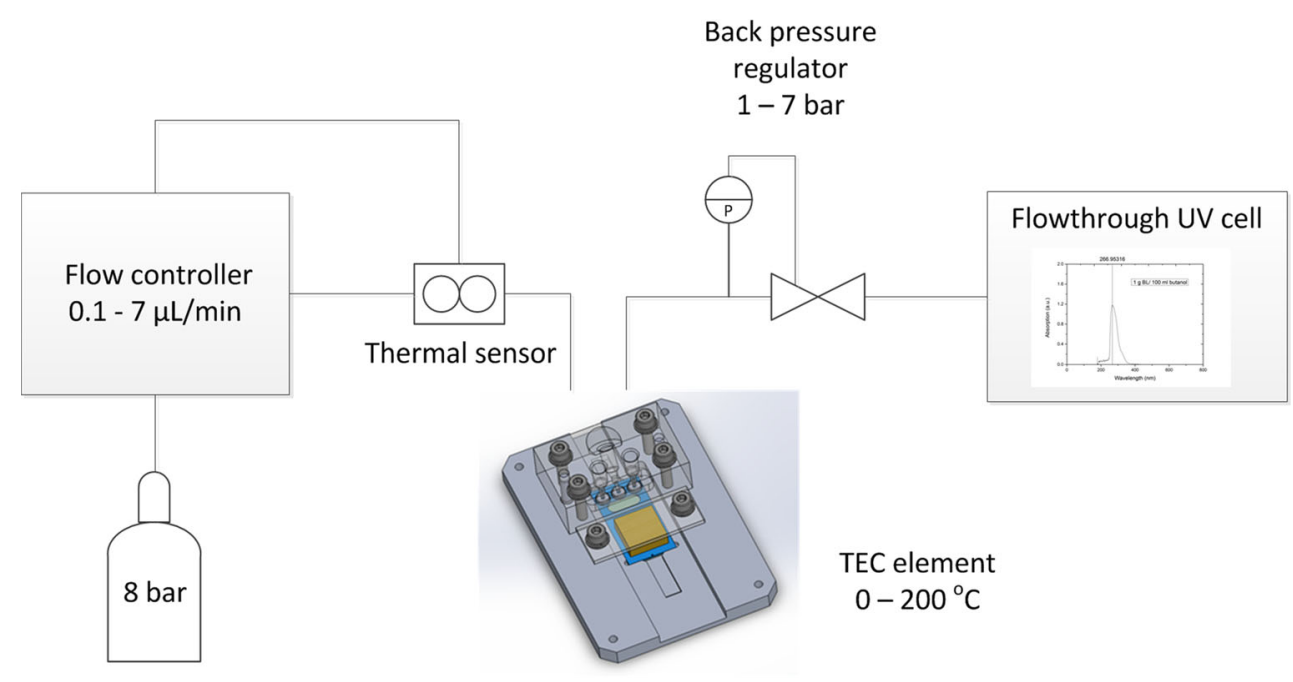

\section{Setup and operation}

The setup allows to independently vary residence time, pressure and temperature while monitoring conversion using inline analysis. The liquid handling system comprises a Fluigent flow controller equipped with a thermal sensor that is connected to a control unit to achieve the flowrate set point. A back-pressure regulator using an active valve controls the pressure, decoupling in this way the flowrate from the pressure. Figure 4 shows the schematic of the set up used. After the pressure reached the set point, the microreactor was heated to the desired temperature. The standard operating conditions for the conversion of fructose to hydroxymethylfurfural (HMF) are 7 bar and $130^{\circ} \mathrm{C}$. The conversion is quantified by measuring the product concentration via inline UV-Vis spectroscopy that tracks the absorption in the UV range of HMF. The maximum absorption peak of HMF is located at $284 \mathrm{~nm}$. The calibration was carried out for different concentrations of HMF solutions (from 2 to $15 \mathrm{mg} / \mathrm{L}$ ). The fitted calibration curve is presented by: $A_{284 n m}=0.1174 \cdot c_{H M F}[\mathrm{mg} / \mathrm{L}]$. The product molar yield was evaluated according to $[h] /[f]_{0}$. 100 , where $[f]_{(0)}$ is the initial fructose concentration and $[h]$ is the HMF concentration.
Fig. 5 a High resolution SEM of a $250 \mathrm{~nm} \mathrm{ZrO}_{2}$ layer. b Higher SEM magnification revealing the dense structure. $\mathbf{c}$ TEM of sputtered $\mathrm{ZrO}_{2}$ displaying its polycrystalline morphology a

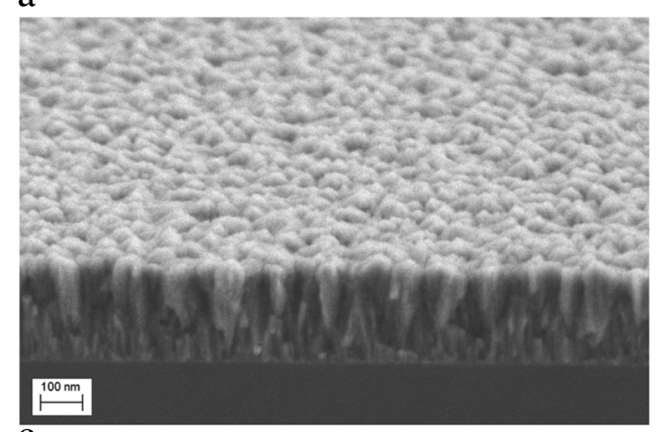

b

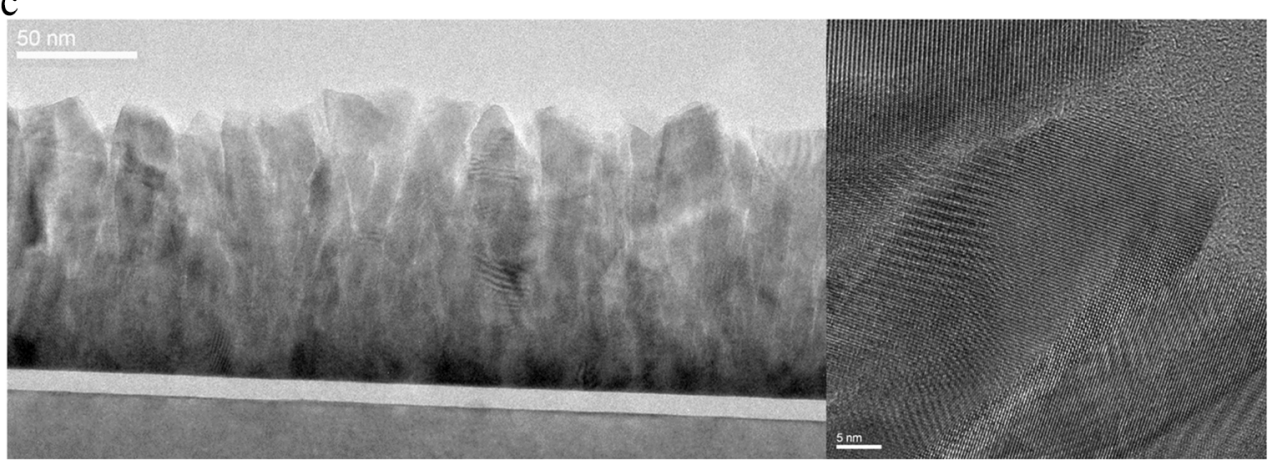




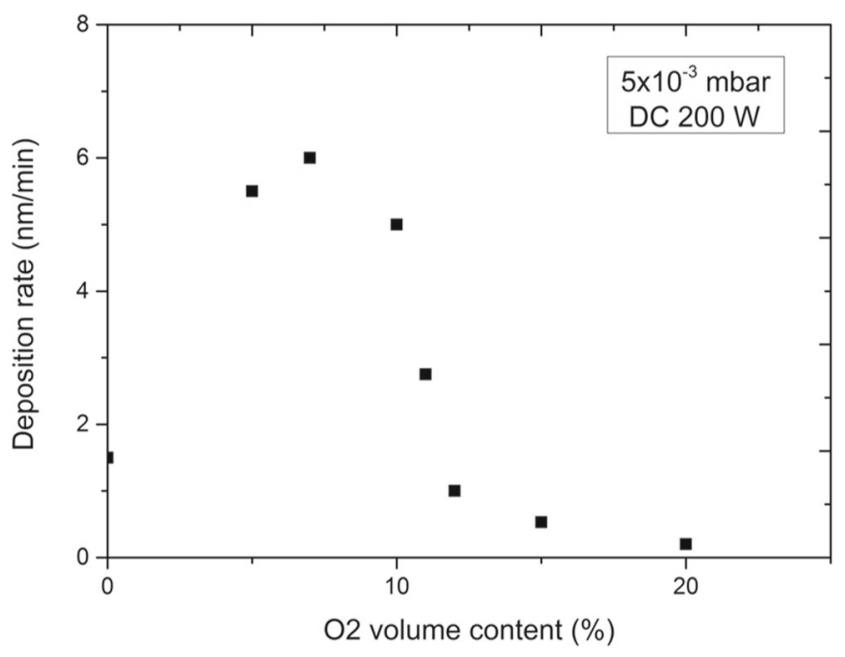

Fig. 6 Deposition rate dependency on volumetric gas composition

\section{Results and discussion}

The sputtered zirconia layer has a dense structured as visualized by $\mathrm{HRSEM}$ (Fig. 5). The 1:2 elemental stoichiometry for $\mathrm{Zr}: \mathrm{O}$ is constant for a wide range of $\mathrm{O}_{2}$ concentrations used during the sputtering process. The elemental content was measured with both XPS and EDX. The observed drop in deposition rate for high $\mathrm{O}_{2}$ concentrations is attributed to the oxidation of the target prior to sputtering (Fig. 6) [24]. For the final recipe, a $7.5 \%$ (vol.) $\mathrm{O}_{2}$ was chosen to ensure a high deposition rate which results in a $250 \mathrm{~nm}$ layer for a $40 \mathrm{~min}$ deposition time as shown in Fig. 5a and b XRD shows a mixture of tetragonal and monoclinic crystalline phases (Figure S2). The annealing process increases the crystallinity of the film, with the previous amorphous phase transitioning to the tetragonal structure which shows up in an increase in the $\mathrm{T}(1,1,1)$ peak intensity. TEM revealed a polycrystalline film

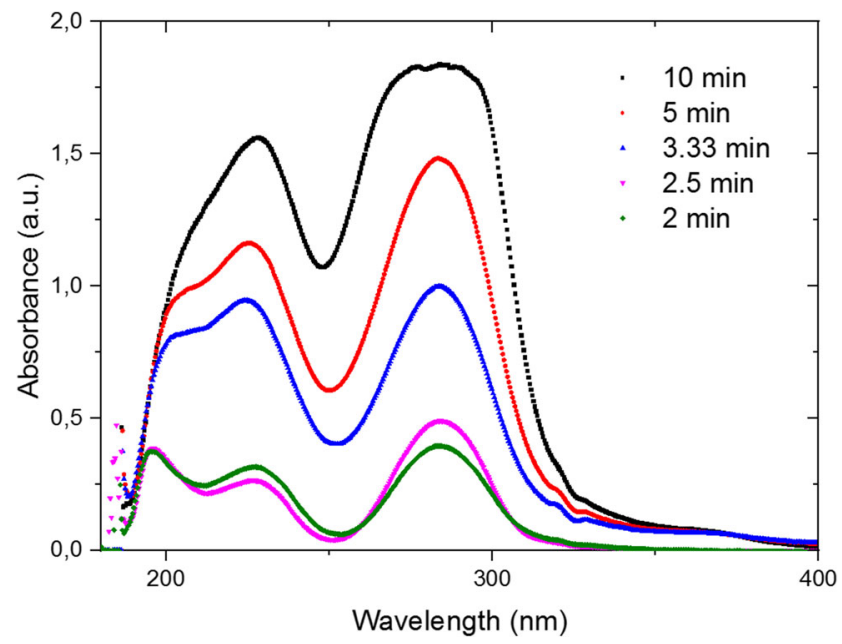

Fig. 8 HMF absorbance showing the increase in HMF production for higher residence times. Reaction performed at $130^{\circ} \mathrm{C}$ and 7 bar using the porous $\mathrm{TiO}_{2}$ layer

(Fig. 5), as sputtering is a high energy process which does not give the possibility for preferential orientation.

The wash coated titania film displays a high degree of homogeneity (Fig. 7). While the initial suspension shows already a narrow size distribution (Figure S3), $157 \pm 70$ $\mathrm{nm}$, there are small aggregates comprising of monodisperse particles of $21 \mathrm{~nm}$ which are the building blocks of the final coating as it is shown in Fig. 7. A porosity of $45 \%$ and roughness similar to the particle radius $(\sim 8 \mathrm{~nm})$ was measured by Spectroscopic Ellipsometry. The specific surface area of the porous film given the particle size and the density of anatase $\left(3895 \mathrm{~kg} / \mathrm{m}^{3}\right)$ is about $50 \mathrm{~m}^{2} / \mathrm{g}$. The crystalline phase is not affected by the sintering process. The $80 \%$ anatase to $20 \%$ rutile composition specified by the manufacturer is preserved. The high quality of the commercial suspension and the constant displacement rate
Fig. 7 High resolution SEM of $\mathrm{TiO}_{2}$ showing. a the porous structure and $\mathbf{b}$ the monodisperse particles. c Cross-section of the wash coated uniform layer
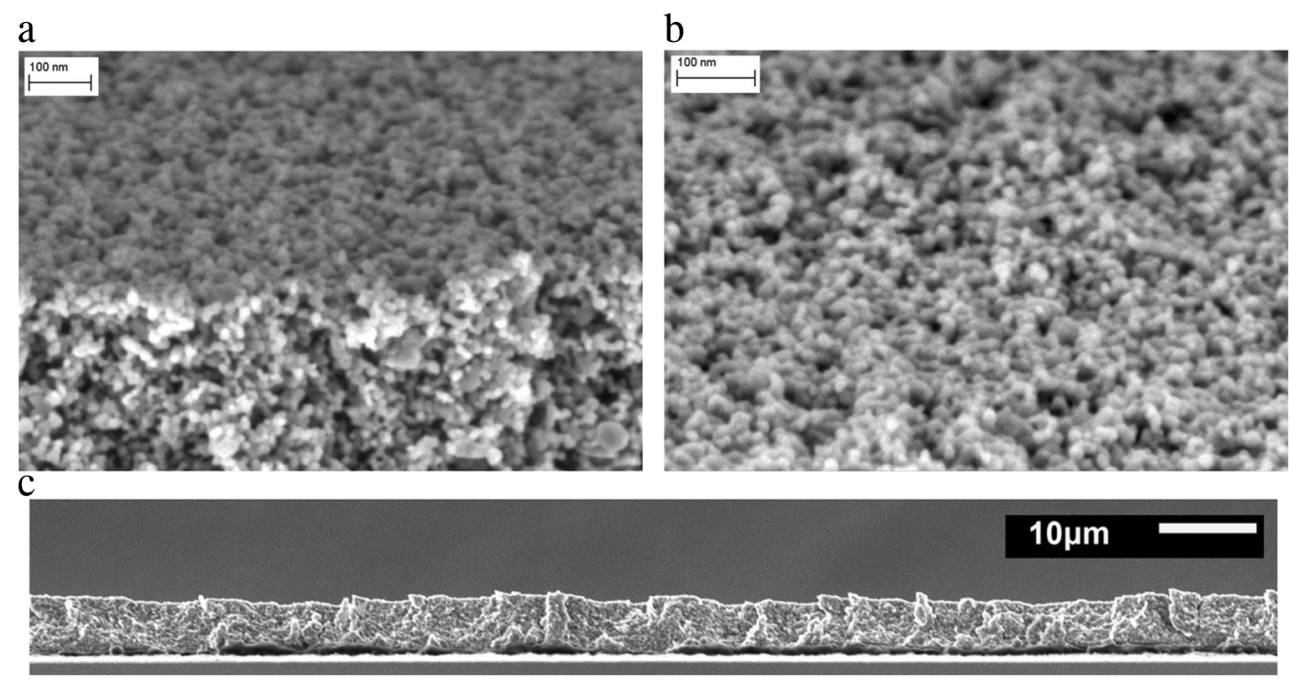
ensured a uniform thickness of the wash coated catalyst which can be observed in the cross section of the channel (Fig. 7c). A displacement rate of $17 \mathrm{~mm} / \mathrm{s}$ led to a thickness of $5 \pm 0.5 \mu \mathrm{m}$.

The reaction was performed at 7 bar and $130{ }^{\circ} \mathrm{C}$ for different flowrates. The product (HMF) absorption peak was monitored at each corresponding residence time (Fig. 8), after steady state was reached. Given the small product yield (Fig. 9), the conversion is reaction rate limited and external mass transfer does not have to be taken into account. This assumption is valid when the second Damköhler number, $D a_{I I}<10^{-1}$, which varies for the fitted reaction constants between $4 \times 10^{-3}$ and $1.3 \times 10^{-2}$.

$D a_{I I}=\sqrt{\frac{k}{D^{0}}} \cdot H$

where $k$ is the volumetric reaction rate constant [1/s] for a first order reaction rate, $H$ is the height of the channel. $D^{0}$ is the diffusion coefficient of fructose in water corrected for the change in temperature and pressure by using the Stokes Einstein equation:

$D^{0}=\frac{k_{B} T}{6 \pi \eta^{0} a}$

$k_{B}$ and $\eta^{0}$ are the Boltzmann constant and the viscosity of pure water at temperature $T$ and the corresponding pressure (7 bar). $a=0.365 \mathrm{~nm}$ is the effective hydrodynamic radius of fructose in water at small concentrations [9]. The variation in $a$ with temperature is small, less than $3 \%$, for the experimental conditions involved. A homogeneous concentration profile can be assumed in the transversal direction which simplifies the system to a plug flow reactor (PFR) model. This gives a steady state $1 \mathrm{D}$ advection reaction balance, where we opt for a first order reaction rate expression for fructose. We will later explain how the intrinsic surface reaction rate constant can be extracted from the fitted volumetric rate constant $k$.

$u \frac{d[f]}{d x}=-k[f]$

with velocity $u[\mathrm{~m} / \mathrm{s}]$ and fructose concentration $[f]$. The velocity $u$ corresponds to the residence time $t=L / u$, with channel length $L$, which gives:

$\ln \frac{[f]}{[f]_{0}}=\ln \frac{[f]_{0}-[h]}{[f]_{0}}=-k t$

$\frac{[h]}{[f]_{0}}=1-e^{-k t}$

We take into account only the reaction pathway to HMF, as the present spectroscopic measurement allows for HMF detection only. Mass spectrometry is required to identify other possible secondary products and quantify the absolute conversion of fructose and its selectivity to HMF.

The sputtered $\mathrm{ZrO}_{2}$ displayed a low activity $(k=1.43 \times$ $10^{-3} 1 / \mathrm{min}$ ) which referenced to the geometric surface area of the film converts to a surface reaction rate constant of $k^{\prime \prime}=1.192 \times 10^{-9} \mathrm{~m} / \mathrm{s}$. The slow overall kinetics can be attributed to the obvious low available surface area, but also to a low density of active sites.

The next attempt tried to improve both aspects, increase the specific surface area and alter the surface functionality for an increase in the number of acid sites. The initial concentration of fructose was lowered to $0.5 \mathrm{~g} / \mathrm{L}$ in the case of $\mathrm{TiO}_{2}$ due to the absorption signal saturation beyond HMF concentrations of $15 \mathrm{mg} / \mathrm{L}$. Figure 8 shows the full spectrum of the steady state for each residence time.

The porous titania layer displayed a higher volumetric rate constant due to the higher available surface area, $k=$ $6.34 \times 10^{-3} 1 / \mathrm{min}$. The extracted value is averaged with respect to the volume of the channel. This value needs to
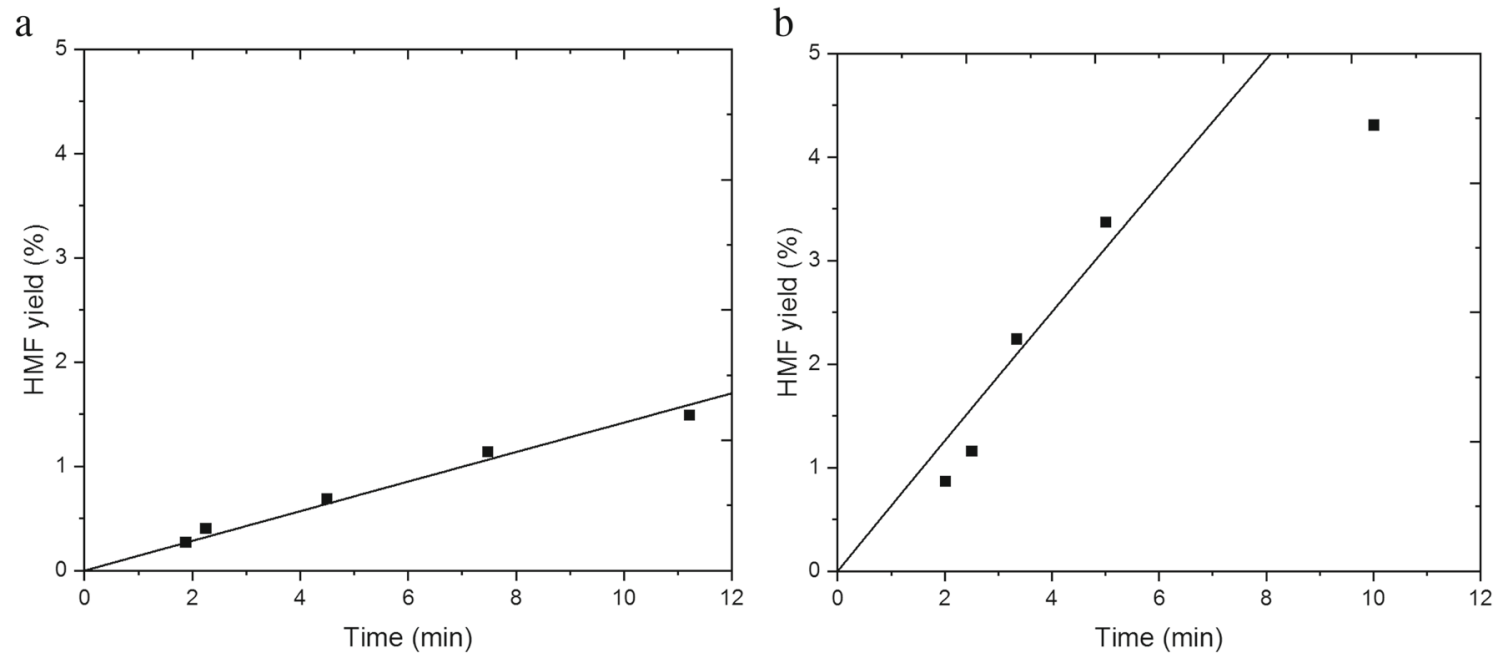

Fig. $9 \mathrm{HMF}$ yield dependence on residence time for a. dense $\mathrm{ZrO}_{2}$ and b. porous $\mathrm{TiO}_{2}$. The data points represent the experimental measurements. The continuous line represents equation 5 where $\mathbf{a} k=1.43 \times 10^{-3} 1 / \mathrm{min}$ and $\mathbf{b} k=6.34 \times 10^{-3} 1 / \mathrm{min}$ 
be rescaled in order to obtain the volume averaged value corresponding to the catalyst layer. The relevant length scales are the height of the channel, $H$, and the catalyst thickness, $\delta$. The following scale relation converts the rate constant per volume of channel to per volume of catalyst: $k_{\mathrm{TiO}_{2}}=k \cdot H / \delta$. To be able to validate the volume averaging of the reaction rate constant, we need to exclude internal mass transfer limitations, such that the catalyst layer is utilized evenly throughout its thickness. Two parameters can be used in this respect: thiele modulus, $\phi$, and the internal effectiveness factor, $\eta$. Thiele modulus evaluates the reaction time scale with respect to the diffusion time scale:

$\phi=\sqrt{\frac{k_{\mathrm{TiO}_{2}}}{D_{\text {eff }}}} \cdot \delta$

Where $D_{\text {eff }}=D^{0} \cdot \frac{\epsilon}{\tau}$ is the effective diffusion coefficient, $\epsilon=0.45$ is the porosity and $\tau=1.35$ is the tortuosity [22]. $\phi$ values up to $6 \times 10^{-3}$ for the extracted kinetics confirmed the reaction driven regime for which the formal criterion is $\phi<10^{-1}$. The effectiveness factor gives the ratio between the net reaction rate and the rate in the absence of concentration and temperature gradients, which for the present system is almost unity:

$\eta=\frac{\tanh \phi}{\phi}$

The conversion from $k_{\mathrm{TiO}_{2}}$ per unit volume $\left[\mathrm{s}^{-1}\right.$ ] to $k^{\prime \prime}$ per unit surface area $[\mathrm{m} / \mathrm{s}]$ can be derived from: $k^{\prime \prime}=$ $k_{\mathrm{TiO}_{2}} /\left(S_{a} \rho(1-\epsilon)\right)$, where $S_{a}=5 \times 10^{-4} \mathrm{~m}^{2} / \mathrm{kg}$ is the specific surface area of $\mathrm{TiO}_{2}, \rho=3895 \mathrm{~kg} / \mathrm{m}^{3}$ is the anatase density and $\epsilon=0.45$ is the porosity. This gave a lower surface reaction rate constant, $k^{\prime \prime}=9.87 \times 10^{-12} \mathrm{~m} / \mathrm{s}$, than the corresponding value for the $\mathrm{ZrO}_{2}$ layer.

The dependency of the reaction rate constant $k$ on temperature is typically expressed by the Arrhenius equation:

$k=k_{0} e^{-E_{a} / R T}$

where $E_{a}$ is the activation energy, $k_{0}$ is the pre-exponential factor and $R=8.31446 \mathrm{Jmol}^{-1} \mathrm{~K}^{-1}$ is the ideal gas constant. We investigated experimentally the dependency of $k$ on temperature for the porous $\mathrm{TiO}_{2}$ layer for which we obtained $E_{a}=80 \mathrm{~kJ} / \mathrm{mol}$ and $k_{0}=e^{19.4} 1 / \mathrm{min}$ (Fig. 10). These values are slightly higher than what Carnity et al. measured for the same reaction using a niobium phosphate catalyst $\left(E_{a}=65.8 \mathrm{~kJ} / \mathrm{mol}\right.$ and $\left.k_{0}=e^{15.7} 1 / \mathrm{min}\right)$ [25]. For these measurements, the surface functionalization procedure using $\mathrm{H}_{3} \mathrm{PO}_{4}$ acid under UV exposure was extended from 4 to $6 \mathrm{~h}$. This change led to an improvement in the reaction rate constant, from $6.34 \times 10^{-3} 1 / \mathrm{min}$ to $11 \times 10^{-3} 1 / \mathrm{min}$ at $130^{\circ} \mathrm{C}$.

While the catalysts investigated in this work displayed rather low activities, the microfluidic platform proved

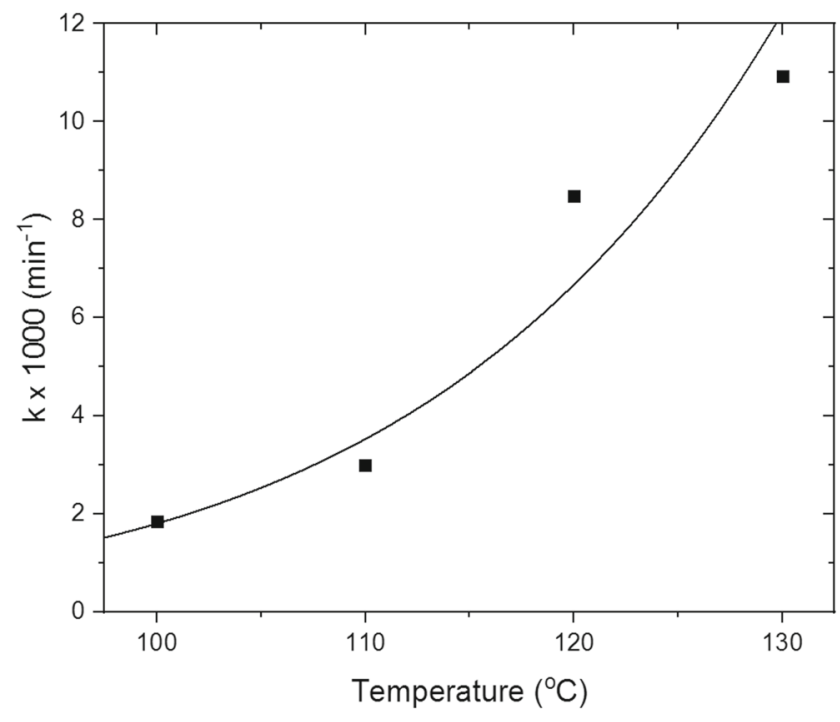

Fig. 10 The dependency of $k$ with temperature. The data points represent the experimental measurements. The continuous line represents Eq. 8 where $E_{a}=80 \mathrm{~kJ} / \mathrm{mol}$ and $k_{0}=e^{19.4} 1 / \mathrm{min}$

to be a useful tool for rapid investigation of catalytic performance. Especially for higher activities when mass transport becomes limiting and accurate modelling is crucial to decouple the kinetics from reactor design, microreactors provide a reliable option due to their well defined fluid dynamics.

\section{Conclusion}

A microfluidic platform was developed for high temperature, high pressure conversion with an inline UV-Vis spectroscopic measurement that facilitates the fast screening of catalytic materials. The well-defined mass transport characteristic for immobilized catalytic layers in microchannels allows for accurate kinetic investigation. The dehydration of fructose to 5-hydroxymethyl-2-furaldehyde (HMF) was studied using both sputtered $\mathrm{ZrO}_{2}$ and wash coated $\mathrm{TiO}_{2}$ layers. The kinetics were determined for each catalyst. For the $\mathrm{TiO}_{2}$ layer, that showed higher conversion, the dependency on temperature was also investigated, revealing an activation energy of $80 \mathrm{~kJ} / \mathrm{mol}$. Surface functionalization of $\mathrm{TiO}_{2}$ using phosphoric acid treatment under UV light proved to increase the catalyst reactivity, likely by enhancing the density of active sites.

Acknowledgments This work was supported by the Netherlands Center for Multiscale Catalytic Energy Conversion (MCEC), an NWO Gravitation programme funded by the Ministry of Education, Culture and Science of the government of the Netherlands. R.G.H.L. also acknowledges the Vici project STW 016.160.312, financed by the Netherlands Organisation for Scientific Research (NWO). 


\section{Compliance with Ethical Standards}

Conflict of interests The authors declare that they have no conflict of interest.

Open Access This article is licensed under a Creative Commons Attribution 4.0 International License, which permits use, sharing, adaptation, distribution and reproduction in any medium or format, as long as you give appropriate credit to the original author(s) and the source, provide a link to the Creative Commons licence, and indicate if changes were made. The images or other third party material in this article are included in the article's Creative Commons licence, unless indicated otherwise in a credit line to the material. If material is not included in the article's Creative Commons licence and your intended use is not permitted by statutory regulation or exceeds the permitted use, you will need to obtain permission directly from the copyright holder. To view a copy of this licence, visit http:// creativecommonshorg/licenses/by/4.0/.

\section{References}

1. Huber GW, Iborra S, Corma A (2006) Chem Rev 106(9):4044. https://doi.org/10.1021/cr068360d

2. Demirbas A (2001) Energy Convers Manag 42:1357. https://doi.org/10.1016/S0196-8904(00)00137-0

3. Luterbacher JS, Martin Alonso D, Dumesic JA (2014) Green Chem 16(12):4816. https://doi.org/10.1039/C4GC01160K

4. de Jong E, Dam MA, Sipos L, Gruter GJM Furandicarboxylic acid (FDCA), a versatile building block for a very interesting class of polyesters, chap 1, pp 1-13. https://doi.org/10.1021/bk-20121105.ch001

5. Vennestrom PNR, Osmundsen CM, Christensen $\mathrm{CH}$, Taarning E (2011) Angewandte Chemie (International ed. in English) 50(45):10502. https://doi.org/10.1002/anie.201102117

6. Du XL, He L, Zhao S, Liu YM, Cao Y, He HY, Fan KN (2011) Angewandte Chemie Int Edn 50(34):7815. https://doi.org/10. 1002/anie. 201100102

7. Tang X, Chen H, Hu L, Hao W, Sun Y, Zeng X, Lin L, Liu S (2014) Appl Catal B: Environ 147:827. https://doi.org/10.1016/j. apcatb.2013.10.021
8. Dusselier M, Mascal M, Sels BF (2014). In: Selective catalysis for renewable feedstocks and chemicals. Springer, Cham, pp 1-40, https://doi.org/10.1007/128-2014-544

9. Lee YC, Chen CT, Chiu YT, Wu KCW (2013) ChemCatChem 5(8):2153. https://doi.org/10.1002/cctc.201300219

10. Saini JK, Saini R, Tewari L (2015) 3 Biotech 5(4):337. https://doi.org/10.1007/s13205-014-0246-5

11. Hattori M, Kamata K, Hara M (2017) Phys Chem Chem Phys 19(5):3688. https://doi.org/10.1039/C6CP06864B

12. De Souza RL, Yu H, Rataboul F, Essayem N (2012) Challenges 3(2):212. https://doi.org/10.3390/challe3020212

13. Du XL, Bi QY, Liu YM, Cao Y, Fan KN (2011) ChemSusChem 4(12):1838. https://doi.org/10.1002/cssc.201100483

14. Yan K, Chen A (2014) Fuel 115:101. https://doi.org/10.1016/j. fuel.2013.06.042

15. Chia M, Dumesic JA (2011) Chem Commun 47(44):12233. https://doi.org/10.1039/c1cc14748j

16. Watanabe M, Aizawa Y, Iida T, Nishimura R, Inomata H (2005) Appl Catal A: General 295(2):150. https://doi.org/10.1016/j. apcata.2005.08.007

17. Nakajima K, Noma R, Kitano M, Hara M (2014) J Mol Catal A: Chem 388-389:100. https://doi.org/10.1016/j.molcata.2013.09.012

18. Ordomsky VV, Sushkevich VL, Schouten JC, Van Der Schaaf J, Nijhuis T (2013) J Catal 300:37. https://doi.org/10.1016/j.jcat. 2012.12.028

19. Pagán-Torres YJ, Wang T, Gallo JMR, Shanks BH, Dumesic J (2012) ACS Catal 2(6):930. https://doi.org/10.1021/cs300192z

20. Zhao H, Holladay JE, Brown H, Zhang ZC (2007) Science 316(5831):1597. https://doi.org/10.1126/science.1141199

21. Siqueira BG, Silva MAP, Moraes C (2013) Brazil J Petrol Gas 7(2):71. https://doi.org/10.5419/bjpg2013-0006

22. Visan A, Rafieian D, Ogieglo W, Lammertink RGH (2014) Appl Catal B: Environ 150-151:93. https://doi.org/10.1016/j.apcatb. 2013.12.003

23. Marre S, Baek J, Park J, Bawendi MG, Jensen KF (2009) J Assoc Lab Autom 14(6):367. https://doi.org/10.1016/j.jala.2009.06.005

24. Depla D, Heirwegh S, Mahieu S, Haemers J, De Gryse R (2007) J Appl Phys 101(1):013301

25. Carniti P, Gervasini A, Biella S, Auroux A (2006) Catal Today 118(3-4):373. https://doi.org/10.1016/j.cattod.2006.07.024

Publisher's note Springer Nature remains neutral with regard to jurisdictional claims in published maps and institutional affiliations. 